\title{
Atom Probe Tomography Characterization of Catalyst Nanoparticles
}

\author{
Paul A. J. Bagot ${ }^{1}$, Tong $\mathrm{Li}^{2}$, Edman Tsang ${ }^{3}$, George D. W. Smith ${ }^{1}$ and Michael P. Moody ${ }^{1}$ \\ 1. Department of Materials, University of Oxford, Parks Road, Oxford OX1 3PH, UK \\ 2. Australian Centre for Microscopy \& Microanalysis, University of Sydney, Australia \\ 3. Chemistry Research Laboratory, Oxford University, 12 Mansfield Road, Oxford OX1 3TA, UK.
}

Catalytic materials play an increasingly critical, yet largely unnoticed, role underpinning countless modern technologies [1]. Many of these catalysts take the form of nanoparticles, discrete structures ranging in size from 1 to 100 nanometres. Catalytic performance can be strongly correlated to size, shape, chemistry and structure of these nanoparticles. To design the next generation of increasingly effective catalysts, researchers urgently require the means for careful characterization of nanoparticles to: improve the quality of their synthesis; gain insight into the optimal nanoparticle properties for a given reaction; and understand fundamental nanoscale processes defining the correlation between a type of nanoparticle and its performance. Atom probe tomography (APT) provides an atom-by-atom reconstructed image of a given material specimen. Each atom is individually chemically identified and their relative positions precisely located in 3D. This type of information is critical to advancing catalyst technology but the application of APT to the analysis of nanoparticles is difficult and we have recently undertaken research to this effect. The results hint at the massive potential of APT, however, there remains limitations to the quality of imaging that we can currently generate.

The specimen shape required by APT poses considerable challenges for the analysis of nanoparticles. One approach is to deposit the nanoparticles upon the surface of a pre-sharpened needle. We have recently demonstrated that not only is it possible to image individual nanoparticles using this approach but APT has the exciting potential to bring invaluable new insights in terms of nanoparticle size, chemistry and structure [2]. However, improved APT results require a more uniform atom-by-atom and layer-by-layer evaporation of ions across the surface than can be achieved in the case for nanoparticles simply deposited onto the specimen. An alternative to the direct deposition of nanoparticles onto the surface is to fabricate APT specimen with the nanoparticles embedded inside. Embedding the nanoparticles should result in a more controlled evaporation, atom-by-atom and tangential to the surface of the specimen, as required by the image reconstruction protocol. In turn, this will minimize the described limitations to the imaging and lead to a markedly improved level of accuracy and detail in the characterization. To this end, we have investigated using advanced prototype beam-sources to embed nanoparticles directly into the substrate. This approach provides precise control over the nanoparticle size, composition and number density, facilitating optimization of the APT analysis. Figure 1 shows an example of this approach, in which individual Ag nanoparticles have been embedded into a Pt matrix support. We will present APT results from such systems together with complementary information from other techniques to probe nanoparticle structure and catalytic performance. 
References:

[1] A. T. Bell, Science, 299, (2003), p. 1688.

[2] K. Tedsree et al., Nature Nanotechnology 6, (2011), p. 302.

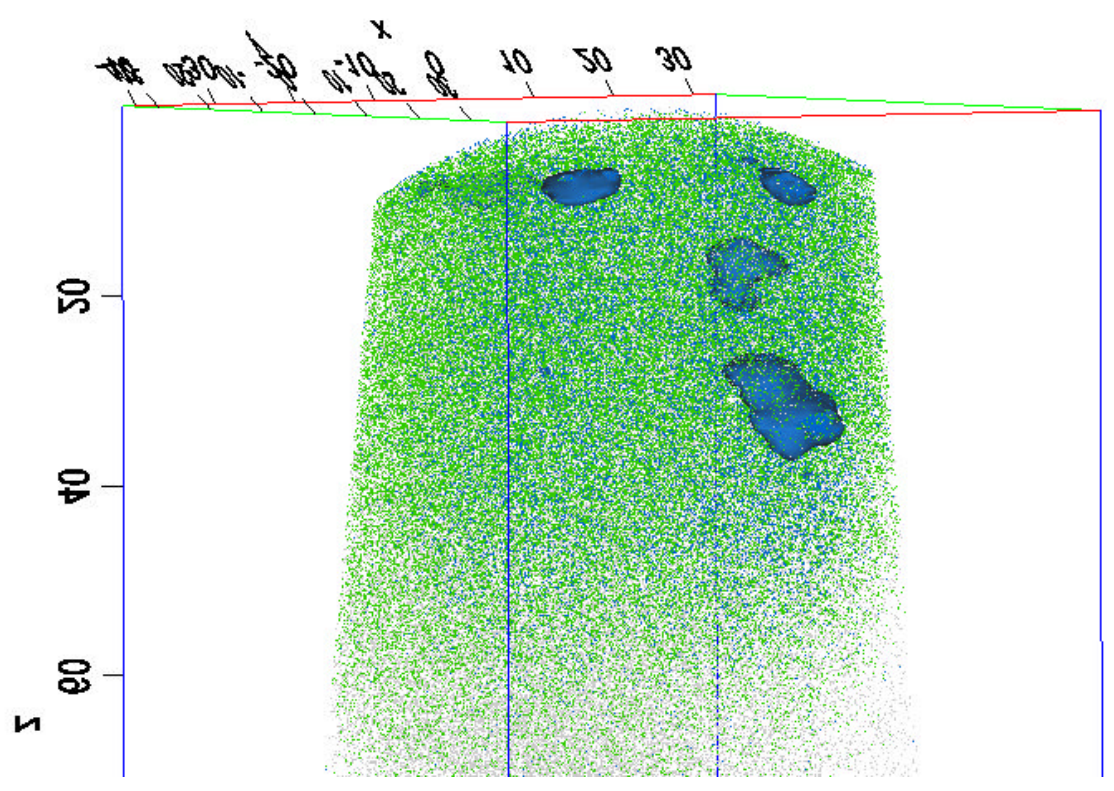

Figure 1. Atom probe reconstruction of $\mathrm{Ag}$ nanoparticles (blue iso-concentration surfaces) encapsulated with Pt support matrix (green) on a silicon post (grey). 